\title{
The Mode Analysis of Sandwich Beam Subjected by Thermal Load Peng Niu ${ }^{1, a^{*}}$ \\ ${ }^{1}$ Institute of architectural engineering , Shenyang University ,Shenyang, 110044, China \\ a444977280@qq.com \\ * The Corresponding Author
}

Keywords: Sandwich beam; Thermal buckling; Elastic foundation; Shooting method; Mode transition

\begin{abstract}
On the basis of accurately considering axial extension and the first-order transverse shearing, geometrically nonlinear governing equations for composite sandwich beams with elastic support, subjected to thermal loads, are formulated. By using a shooting method, the two-point boundary value problem for nonlinear ordinary differential equations are solved numerically and the numerical solutions to static thermal post-buckling and thermal bending deformation of the sandwich beams with pinned-fixed ends under transverse uniform thermal loads are obtained. Characteristic curves of the critical buckling temperature versus foundation stiffness parameter for the first three buckling modes are plotted and critical thermal buckling modes transition feature are given.
\end{abstract}

\section{Introduction}

Thermal expansion along axial direction under temperature load leads to thermal post-buckling deformation of beams and rods, which is required to be studied with axial extension. Measurement of stability of axially extensible beams has been one basic calculation problem in physics, and one subject of interest to researchers ${ }^{[1-6]}$. Many studies have been done of elastic beams and panels. $\mathrm{Li}^{[7]}$ explored displacement and angular rotation of beams on a Winkler foundation under randomly distributed mechanical load. Zhang ${ }^{[8]}$ studied the elastic beams on a non-linear foundation subjected to a longitudinal force, and different mode transition caused by different mechanical load. Pate ${ }^{[9]}$ numerically analyzed the effect of nonlinear free bending vibration and transverse shearing deformations on beams on a two-parameter elastic foundation. Shen ${ }^{[10,11]}$ examined the effect of post-buckling on composite panels under mechanical and thermal load by using Galerkin method. $\mathrm{Li}^{[12]}$ studied, based on plate theory, post-buckling and mode transition of elastic circular panel on a Winkler foundation subjected to uniform temperature rise.

Figure 1. Finite Geometry of sandwich beam under elastic foundation and rotational springs

Literature survey shows that there are still few researches into the effect of stiffness change on
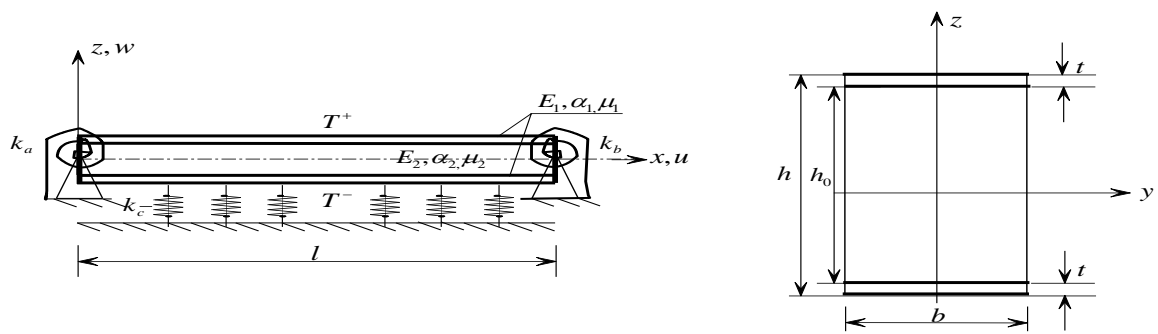

thermal buckling and thermal post-buckling behaviors of composite sandwich beams on an elastic foundation under temperature load. In this paper, based on axial extension and first-order shear deformation theory ${ }^{[3,12]}$, governing equations for nonlinear thermal buckling of composite sandwich beams subjected to temperature load on an elastic foundation are derived. With the shooting method, numerical solutions for thermal bending and buckling of a sandwich beam under uniform transverse temperature rise are obtained. This paper highlights mode transition caused by foundation stiffness 
change, to which the corresponding stiffness and critical temperature are presented.

\section{Governing Equation}

Supposing there is a composite sandwich straight beam with rectangular section, its length, width and height are $1, b$ and $h_{0}$, respectively (see table 1 ). Modulus of elasticity, coefficient of expansion, and Poisson's ratio of the panel and the core are given in Figure 1a. Made up of same material, both the upper and lower panels are $\mathrm{t}$ in thickness; therefore, the core is $\mathrm{h}=\mathrm{h}_{0}-2 \mathrm{t}$ in thickness. The beam has a rectangular section, as shows in Figure 1b. Assuming that the beam is subjected to a temperature rise which changes with thickness, the temperature rises, a transverse linear rise, in the upper and lower panels are $\mathrm{T}^{+}$and $\mathrm{T}^{-}$, respectively. It is assumed that physical parameters of different layers of the beam do not change with the rise of the temperature. The beam, at the bottom, is supported by an elastic foundation, which is $K_{c}$ in stiffness, and in both ends, is restrained by two movable springs, which are ka and $\mathrm{kb}$ in stiffness, respectively. The static response of thermal buckling of the beam was analyzed, with axial extension and transverse shear deformation taken into consideration. Its corresponding dimensionless governing equation is given below ${ }^{[3]}$ :

$$
\begin{aligned}
& \frac{\mathrm{d} S}{\mathrm{~d} \xi}=\Lambda_{0}, \frac{\mathrm{d} U}{\mathrm{~d} \xi}=\Lambda_{0} \cos \theta-1, \frac{\mathrm{d} W}{\mathrm{~d} \xi}=\Lambda_{0} \sin \theta, \frac{\mathrm{d} \varphi}{\mathrm{d} \xi}=m+m_{T} \\
& \frac{\mathrm{d} P_{H}}{\mathrm{~d} \xi}=0, \frac{\mathrm{d} P_{V}}{\mathrm{~d} \xi}=\Lambda_{0} W K_{c}, \frac{\mathrm{d} m}{\mathrm{~d} \xi}=\Lambda_{0}\left(P_{H} \sin \theta-P_{v} \cos \theta\right) \\
& \Lambda_{0} \cos \theta=\Lambda_{0} \cos (\varphi-\gamma)=D_{1} \cos \varphi+D_{2} \sin \varphi, \Lambda_{0} \sin \theta=\Lambda_{0} \sin (\varphi-\gamma)=D_{1} \sin \varphi-D_{2} \cos \varphi \\
& D_{1}=\frac{\left(P_{H} \cos \varphi+P_{V} \sin \varphi+P_{T}\right)}{F_{1}}+1, D_{2}=\frac{\beta\left(P_{H} \sin \varphi-P_{v} \cos \varphi\right) \phi_{3}}{\left[12 \delta^{2}\left(c+(1-c) K_{1}\right)\right]}, \Lambda_{0}=\sqrt{D_{1}^{2}+D_{2}^{2}}, \tan \gamma=D_{2} / D_{1} \\
& P_{T}=\tau_{m} \eta_{1} / \phi_{3}, m_{T}=\tau_{d} \eta_{2} /\left(12 \delta \phi_{3}\right), F_{1}=12 \delta^{2} \phi_{1} / \phi_{3}, F_{4}=12 \delta^{2} \eta_{3} / \phi_{3} \\
& \text { The dimensionless boundary conditions are as follows: } \\
& S(0)=0, U(0)=0, W(0)=0 \quad \varphi(0) \beta_{0}, m(0)=K_{a} \varphi(0) \\
& \\
& U(1)=0, W(1)=0, \quad m(1)=-K_{b} \varphi(1)
\end{aligned}
$$

\section{Numerical Calculation and Results Analysis}

Eqs.(1) and (2) are nonlinear ordinary equations with boundary value problems to be solved; therefore, they are hard to be solved with the analytical method. In this paper, with the shooting method ${ }^{[3-6]}$, numerical solutions are obtained. When calculating, assume that the upper and lower layer are made up of metal while the middle of core.

As an example, a beam constrained by an elastic foundation but not by rotational springs is considered. Assuming that the end constraints of the beam are not symmetrical, i.e. there is unmovable hinge supports on the left side while a fixed pin on the right. The effects of other forms of elastic restraint on the stability of beams are further discussed in the subsequent paper. The characteristics of buckling and post-buckling deformation of composite sandwich beams subjected to uniform temperature rise are analyzed when $c=0.1$, and $\delta=30$. 


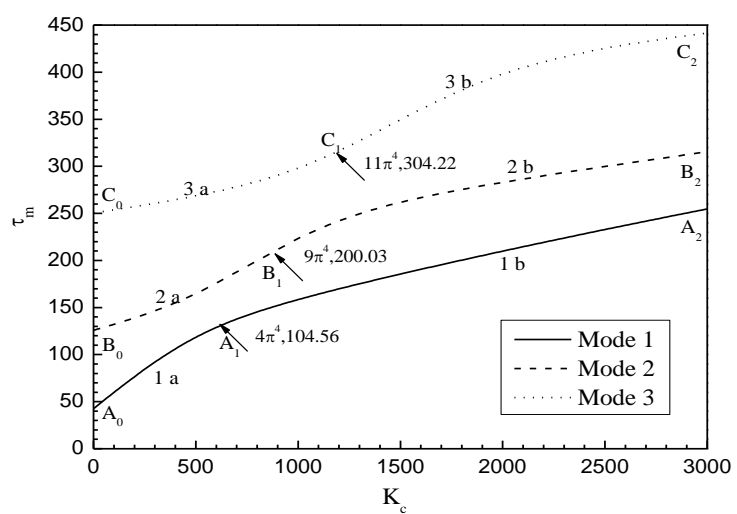

Figure 2. Finite Characteristic curves of the $\tau_{c r}$ versus $K_{c}$ for the first three buckling modes

In Fig. 2, the relationship curve between buckling temperature and foundation stiffness of composite sandwich beams is given when there is unmovable hinge supports on the left side while a fixed-pin on the right. Three relationship curves without cross-over points are given when $K_{c} \leq 3000$. Three buckling modes corresponding to three buckling temperature points are presented when $K_{c}=0$. There is one special point in each curve, i.e. the stiffness point for mode transition, and it divides each curve into two parts. In the figure, three stiffness points, $A_{1}, B_{1}, C_{1}$, are showed, and their positions are $\left(K_{c}, \tau_{m}\right)_{1}=\left(4 \pi^{4}, 105.5610\right), \quad\left(K_{c}, \tau_{m}\right)_{2}=\left(9 \pi^{4}, 200.03\right)$ and $\left(K_{c}, \tau_{m}\right)_{3}=\left(11 \pi^{4}, 304.22\right)$, respectively. Near these stiffness points, mode transition takes place in sandwich beams. Whether there is a change of polarity of moment of flexure, or whether the curvature is zero is also our basis for the determination of the occurrence of mode transition.

Fig 3 shows the buckling mode change which is corresponding to transition stiffness and the nearby critical temperature in Fig. 2. To be specific, $A_{0} A_{1}$, and $A_{1} A_{2}$ in Fig 2 are corresponding to mode 1a (first-order) and mode 1b (second-order) in Fig 3. These two modes are critical buckling modes. When an elastic foundation stiffness parameter $K_{c}$ is given, buckling mode which is corresponding to the minimum buckling temperature is the critical buckling mode. $B_{0} B_{1}$ and $B_{1} B_{2}$ in Fig. 2 are corresponding to mode $2 \mathrm{a}$ (second-order) and mode $2 \mathrm{~b}$ (third-order) in Fig. 3. $C_{0} C_{1}$ and $C_{1} C_{2}$ are corresponding to mode $3 \mathrm{a}$ (third-order) and mode $3 \mathrm{~b}$ (fourth-order) in Fig. 3. All these four modes are non-critical buckling modes.

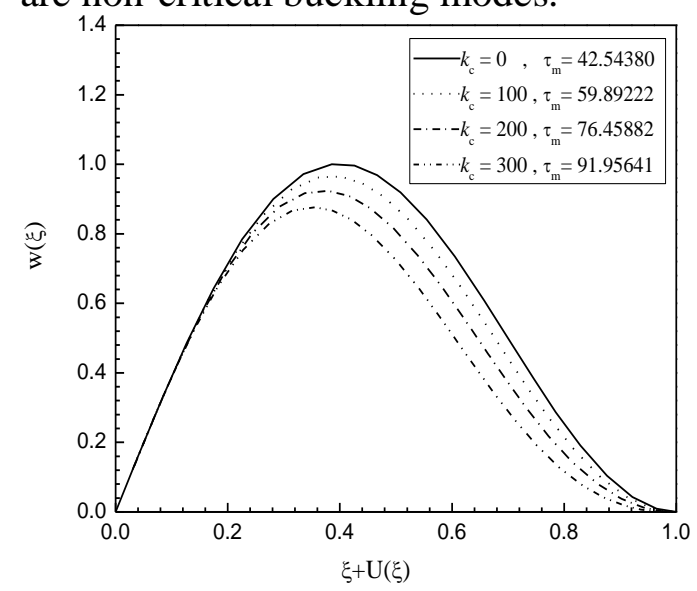

(a)The first critical buckling mode (1a)

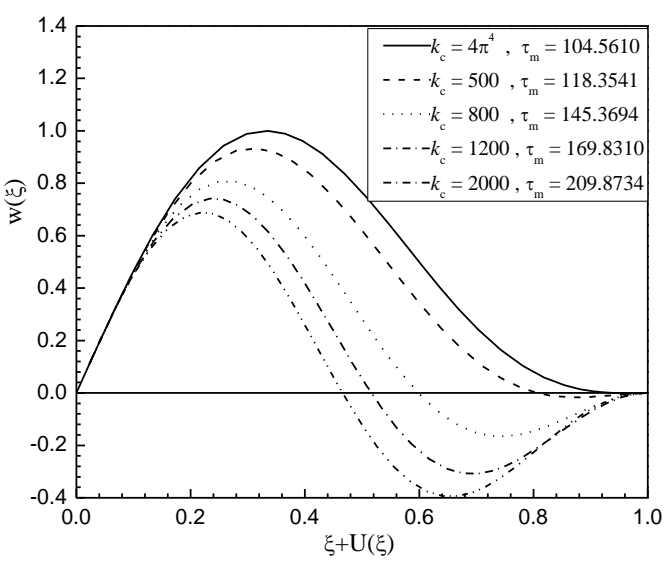

(b)The second critical buckling mode (1b) 


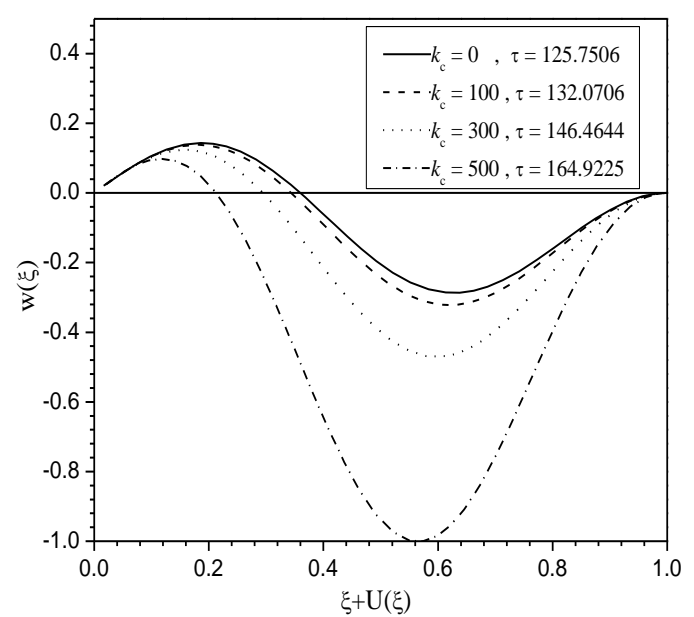

(c)The second non-critical buckling mode (2a)

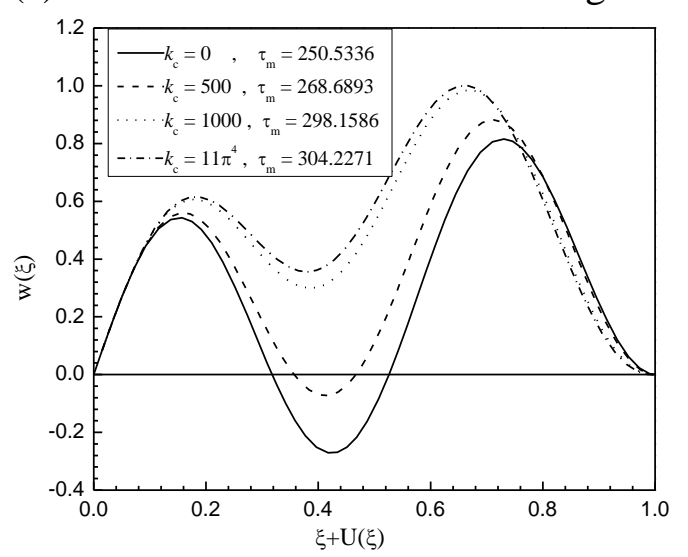

(e)The third non-critical buckling mode(3a)

Figure 3. Finite Critical buckling mode shapes of the sandwich beam for some specified values of

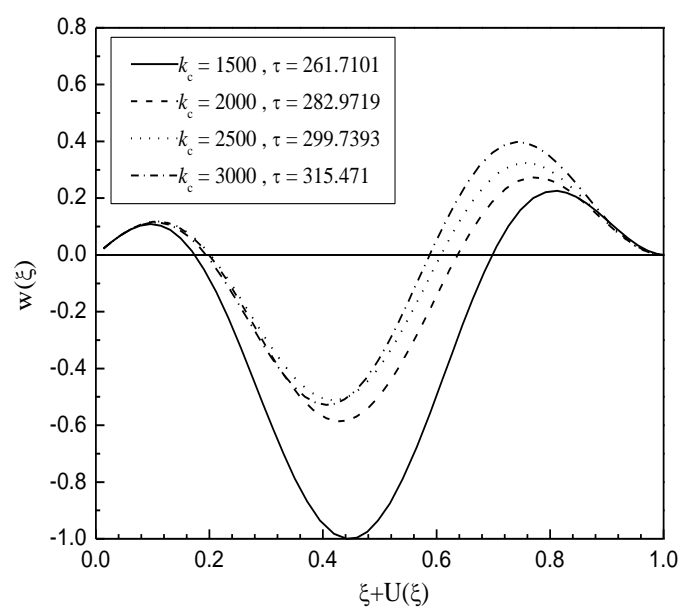

(d)The third non-critical buckling mode (2b)

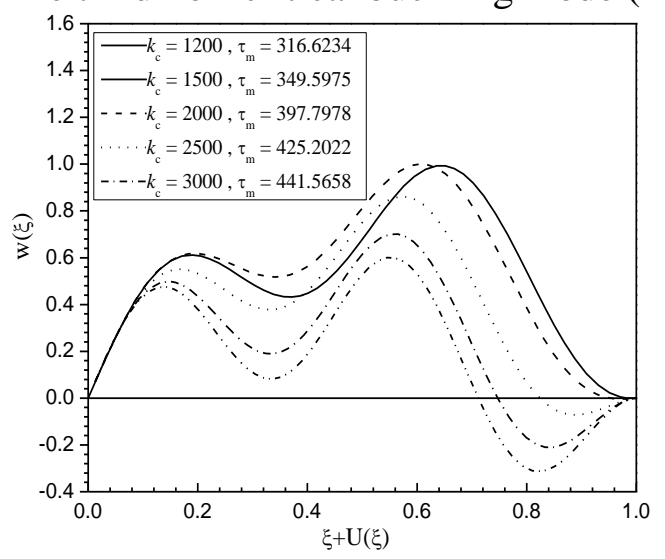
(f)The fourth non-critical buckling mode (3b)
sandwich beam for some specified values of $K_{c}$

When $K_{c}=100$ and $K_{c}=1000$, different rotational angles on the left side and dimensionless temperature are corresponding to different post-buckling equilibrium configurations (see Fig. 4). With the rise of the dimensionless temperature, the deformation of beams increases, which is in agreement with common physical phenomenon. When beams are in buckling state, modes of different orders do not vary with the change of temperature. Even though the configurations vary in scale after deformation, their shape never changes.
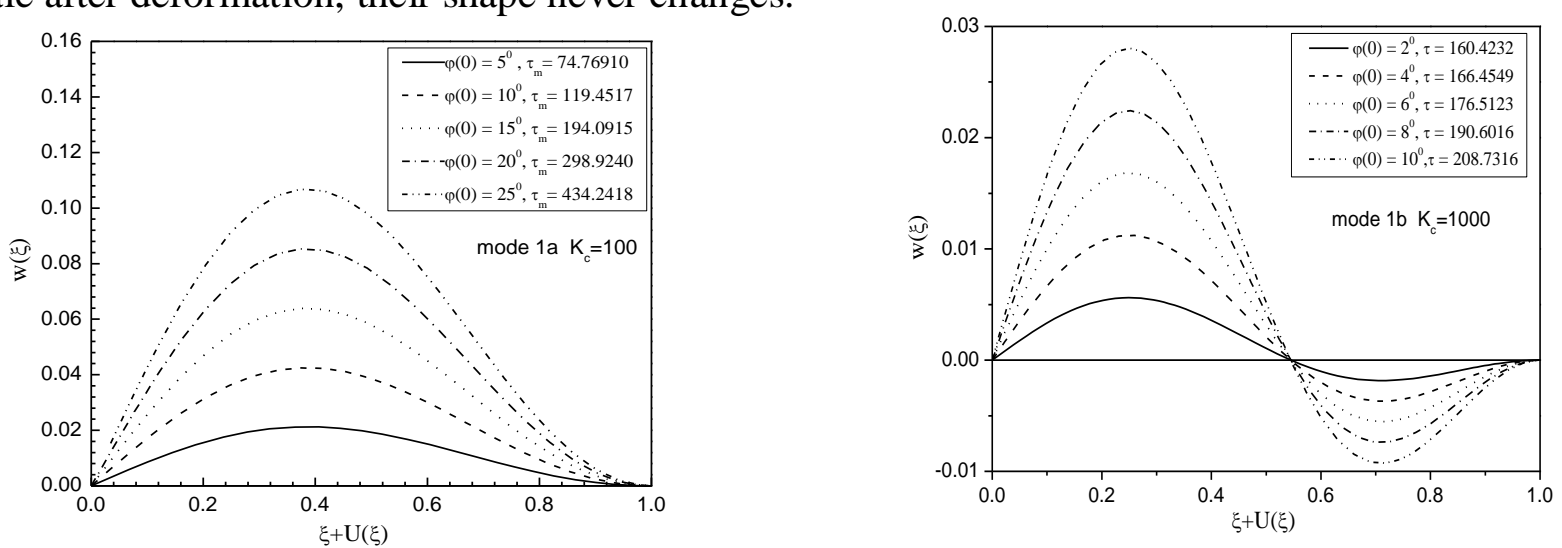

Figure 4. Finite Thermal post-buckling equilibrium configurations of the beam in the first mode for the different values of $\varphi(0)$ and $\tau_{m}$ when $K_{c}=100,1000$ 


\section{Conclusion}

Based on axial extension and first-order shear deformation theory, the numerical solutions of boundary value problems of ordinary equations are obtained with the shooting method. The buckling and post-buckling characteristics of sandwich beams on an elastic foundation are also discussed with emphasis on the effect of foundation stiffness on critical buckling state. The major findings are as follows:

(1)For the first three kinds of critical buckling modes, critical temperature monotonically rises with the increase of foundation stiffness. The three curves have no cross-over points, but each has a mode transition point. The transition stiffness and critical temperature corresponding to these three points are $\left(K_{c}, \tau_{m}\right)_{1}=\left(4 \pi^{4}, 105.5610\right),\left(K_{c}, \tau_{m}\right)_{2}=\left(9 \pi^{4}, 200.03\right)$, and $\left(K_{c}, \tau_{m}\right)_{3}=\left(11 \pi^{4}, 304.2271\right)$. Different transition stiffness values represent the transition characteristics of different modes.

(2)With the rise of the dimensionless temperature, the deformation of beams increases, which is in agreement with common physical phenomenon. When beams are in buckling state, modes of different orders do not vary with the change of temperature. Even though the configurations vary in scale after deformation, their shape never changes.

\section{Acknowledgements}

This work was financially supported by Program for Liaoning Excellent Talents in University(Project No. LJQ2015076), and the National Science and Technology Supporting Plan(Project No.2011BAJ06B04).

\section{References}

[1] D. W. Coffin, F. Bloom.International Journal of Non-Linear Mechanics,34(1999): 935-947.

[2] M. A. Vaz , R. F. Solano. Journal of Thermal stresses, 27 (2004):367-380.

[3] P.Obara. Archives of Civil Engineering.,60(2014)No. 4:50-56.

[4] V Dona; M. G Rajendran. International Conference on Recent Trends In Engineering Science And Management,(2015)

[5] S Jin. Architectural research.16(2014) No.2:75-80.

[6] P Niu, S. R. Li, C. F. Jin, G Yang. Acta Mechanica Solida Sinica, 32(2011)No.5:483-489. (in Chinese)

[7] S.Q.Li, R.B.Zhao, Q.Lu, etc. Journal of Liaoning Technical University (Natural Science), 28(2009)No. 4:558-561. (in Chinese)

[8] Peng L, Shizhao N, Zheng W, Ziwei J, Jianwu Y, Zhongxiang Q, Wangmo P. Predicting durations of online collective actions based on Peaks' heights [J]. Communications in Nonlinear Science and Numerical Simulation. 2018, 55: 338-354.

[9] Y Zhang, K. D. Murphy. Secondary buckling and tertiary states of a beam on a non-linear elastic foundation . Non-Linear Mechanics, (2005) No.40:795-805.

[10] B. P. Patel, M Ganapathi, Touratier M. Composite Structures, (1999)No.46:189-196.

[11] H. S. Shen. Composite Structures, (1999)No.45:115-123.

[12] H. S. Shen. Non-Linear Mechanics, 32(1997) No.6:1035-1050.

[13] S. R. Li ,W. S. Yu. Engineering mechanics, 24(2007) No.5:63-66.(in Chinese) 